\section{A new constraint to improve the prediction of zircon-melt REE partition coefficients.}

\section{LINUS B. STREICHER ${ }^{1}$, WIM VAN WESTRENEN ${ }^{1}$, JOHN M. HANCHAR ${ }^{2}$ AND FRAUKJE M. BROUWER $^{1}$}

${ }^{1}$ Vrije Universiteit Amsterdam

${ }^{2}$ Memorial University of Newfoundland

Presenting Author: linus.streicher@vu.nl

Zircon has many applications in geology due to its physical durability and chemical stability, when crystalline, and occurrence in numerous rock types. Beyond isotopic age determination, several methods for tracing a variety of geological processes have been developed in recent years based on trace element abundances, and radiogenic tracer isotopes (e.g., Lu-Hf) in zircon. Application of these techniques generally requires a quantitative knowledge of the partition coefficients (D) for trace elements between zircon and co-existing bulk rock, fluid, or other minerals. However, determining $\mathrm{D}_{\mathrm{REE}}$ of zircons is challenging. This is especially true for light rare earth elements (LREE) and other incompatible elements, which generally occur in very low concentrations in zircon. As proposed by Hanchar and Westrenen [1], for REE this difficulty can be overcome by modeling $\mathrm{D}_{\mathrm{REE}}$ using a prediction function based on the crystal lattice strain model [2]. The prediction function is based on three empirically derived parameters: the apparent Young's Modulus $E$ of the zirconium site in which the REE reside, the radius of a (fictive) REE optimally fitting into this site $r_{0}$, and the maximum partition coefficient $D_{0}$ of the cation with $r_{0}$. In principle, the three parameters can be determined by fitting the model function to experimentally determined $\mathrm{D}_{\mathrm{REE}}$ using non-linear regression. However, the radii of all REE are all larger than $r_{0}$ for zircon, regularly leading to strain model fits yielding physically unrealistic values of $r_{0}$ in particular (Figure). Here we use an updated compilation of literature zircon-melt REE + Sc partition coefficients to establish a new empirical relationship between $r_{0}$ and $E$ which is used as an extra constraint during lattice strain model fitting. This leads to improved assessments of the $E, D_{0}$, and $r_{0}$ of literature datasets that can also better identify anomalies in D determinations for the LREE (Figure). This in turn, enables the development of improved predictive models that link zircon trace element contents to key geological parameters such as pressure and temperature.

[1] Hanchar \& Westrenen (2007), Elements 3, 37-42

[2] Blundy \& Wood (1994), Nature 372, 452-454

[3] Taylor et al. (2015), J. Metamorph. Geol. 33, 231-48

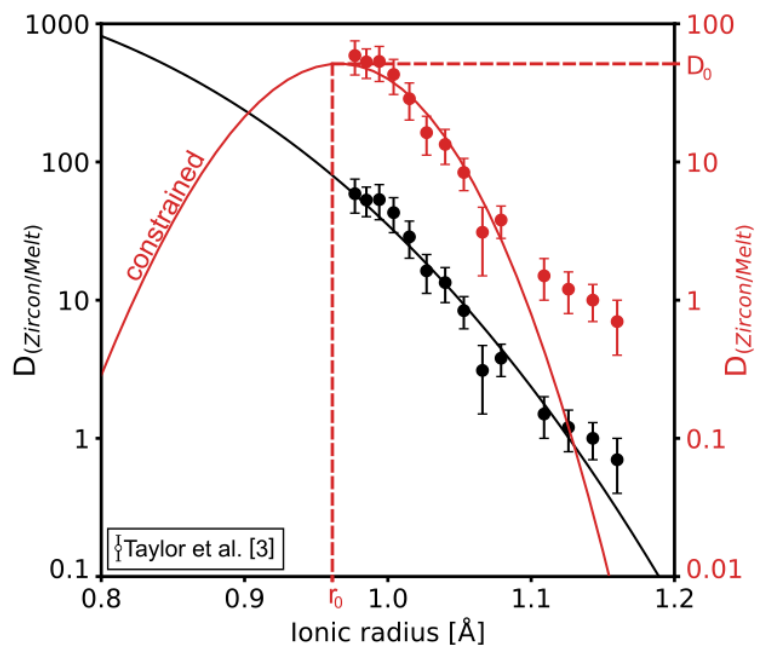

